\title{
A Solar Paradox Resolved
}

\author{
A new model of light-matter interactions solves a decades-old problem by \\ reconciling theoretical predictions and experimental observations of \\ polarized light from the Sun.
}

By Marric Stephens

F or over 20 years, physicists have grappled with a paradox: Despite evidence that the Sun's atmosphere is strongly magnetized, the linear polarization of sunlight at the frequency of one of its absorption lines could only be explained with models that contain magnetic fields no stronger than a few milligauss. A team at the Istituto Ricerche Solari Locarno in Switzerland and the Instituto de Astrofísica de Canarias in Spain has now resolved this conflict by applying a recently developed theory of atom-photon interactions [1]. Their model reproduces the enigmatic polarization signal in the presence of the Sun's gauss-strength magnetic fields.

Most of the light that reaches us from the quiet regions of the Sun's atmosphere (outside of sunspots) is linearly polarized. In the Sun's absorption lines, this polarization occurs because the Sun's anisotropic radiation field induces in the atmosphere's atoms "atomic-level polarization"-imbalances in the populations of the atomic energy sublevels. Until now, the polarization of one particular spectral feature-sodium's $D_{1}$ absorption line-could only be explained if the ground level of

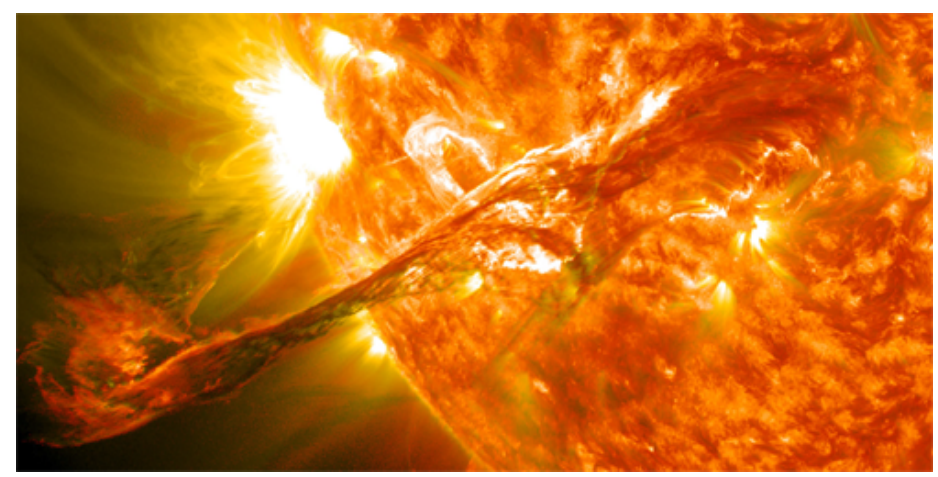

Credit: NASA/GSFC/SDO sodium atoms in the atmosphere was substantially polarized. However, solar magnetic fields were predicted to destroy this ground-level polarization before the atoms could undergo the transition responsible for the spectral feature.

In their new model, the researchers apply a recently developed theory of polarized radiative transfer that, unlike earlier treatments, accounts for spectral variations in light intensity across the hyperfine-structure transitions of the $D_{1}$ absorption line. By including these tiny variations (rather than assuming a uniform spectral intensity), the model can reproduce the observed polarization of the $D_{1}$ line even without ground-level polarization. This resolution to the paradox, the researchers say, will allow new investigations into the complex magnetism of the solar atmosphere.

Marric Stephens is a Corresponding Editor for Physics based in Bristol, UK.

\section{REFERENCES}

1. E. Alsina Ballester et al., "Solving the paradox of the solar sodium- $D_{1}$-line polarization," Phys. Rev. Lett. 127, 081101 (2021). 